\title{
EVALUACIÓN DE LA PRECIPITACIÓN DISTRIBUIDA EN LA CUENCA KATARI BASADO EN TECNOLOGÍA SATELITAL Y PRODUCTOS DERIVADOS
}

\author{
Jhonatan E. Ureña, Andrés G. Vallejos, Oliver C. Saavedra y Ana C. Escalera
}

\section{RESUMEN}

La medición de la precipitación espacial, en alta resolución, es una variable crucial en la modelación hidrológica de cuencas, pero debido a las limitantes con respecto a la cantidad y distribución de pluviómetros, se hace necesario la generación de datos utilizando productos satelitales de precipitación. Para este estudio se usaron los productos: GSMaP, CHIRPS y GMET, cada uno con una resolución espacial y temporal diferente. El área de estudio es la cuenca Katari, al noreste de la ciudad del Alto y presenta alturas entre los 3800 a 5200 m.s.n.m. El periodo temporal seleccionado fue 2000 - 2016 y pare ello se realizó un análisis comparativo divido en subcuencas, donde se emplearon dos niveles comparativos: una comparación entre productos de precipitación base (GSMaP, CHIRPS y SENAMHI) y una comparación entre "GS-Katari”, un producto combinado por el método de ajuste iterativo entre GSMaP y SENAMHI, y “GMET”, un producto combinado a través de métodos estocásticos entre SENAMHI y CHIRPS. Inicialmente se demostró que, tanto GSMaP como CHIRPS presentan una sobrestimación de sus intensidades de precipitación con respecto a los datos de tierra, sin embargo, aparentemente presentan una excepción al acercarse al Lago Titicaca. Posteriormente, se realizó 5 iteraciones de ajuste para obtener el producto combinado GS-Katari, este producto ajustado presenta intensidades de precipitación que tienden a la subestimación con rangos menores al $10 \%$ del valor medido en tierra. Posterior a la generación del producto, se analizó los datos de precipitación entre GMET y el producto combinado y se comprobó que GMET presenta una sobrestimación con respecto a SENAMHI, pero en el área cercana al Lago Titicaca, se observa que GMET presenta una subestimación similar a CHIRPS y GSMaP con respecto a las intensidades de SENAMHI. Los resultados del presente artículo buscan proporcionar datos con una mayor resolución espacial e intensidades de precipitación más procesadas con el fin de generar modelos hidrológicos con menos incertidumbres, con el fin de gestionar de mejor manera los recursos hídricos para la zona.

Palabras Clave: Cuenca Katari, GSMaP, CHIRPS, GMET, Productos de Precipitación Satelital.

DOI: $10.23881 /$ idupbo.018.1-3i 